\title{
Feasibility and Safety of Laparoscopic Resection of Gastro- intestinal Stromal Tumors
}

\author{
Tamer A.El-Bakary, MD; Sherif A. Mustafa, MD.
}

Department of General Surgery Tanta University.

Background: Surgery is still the main stay in treatment of gastro-intestinal (GIST) tumors. Laparoscopic resection should be considered in these cases as their biological behavior doesn't require large margins of excision or extensive lymph nodes dissection.

Patients and methods: A retrospective analysis of 15 patients who underwent laparoscopic resection of GIST. We reviewed the demographics of the patients, diagnostic tools, operative details, and post-operative course.

Results: The mean age of the patients was 49 years. Fifty three \% were males and $47 \%$ were females. The main symptoms were abdominal pain, anemia, and nausea and/or vomiting. GIST was in the stomach in 9 patients and in the small intestine in 6 patients. The mean size of gastric GIST was $4.4 \mathrm{~cm}$ and the mean size of small intestinal GIST was $4 \mathrm{~cm}$. Laparoscopy was successfully done in 14 patients. Only 1 conversion was needed in gastric GIST to control bleeding from short gastric vessels. No tumor rupture occurred. The mean operative time was 118.4 minutes and margins of excision were negative in all the cases. Fifty three \% were very low risk, 27\% were low risk, 7\% were intermediate risk, and 13\% were high risk. Post-operatively, mean time to pass flatus was 1.6 days. Mean time to tolerate full oral intake was 3.8 days. Mean number of narcotic doses was 2.73. Mean length of hospital stay was 4.9 days. One patient had post-operative atelectasis, and 1 patient had superficial umbilical wound infection. During a mean follow up period of 12.6 months, no recurrence or metastasis could be detected.

Conclusion: Laparoscopic resection of GIST is a safe and feasible technique that gives the patient all the advantages of minimally invasive surgery without compromising the oncological outcomes.

Key words: Gastro-intestinal stromal tumor, laparoscopy, resection.

\section{Introduction:}

Gastro-intestinal stromal tumors (GISTs) are the commonest mesenchymal tumors of the intestinal tract. ${ }^{1}$ GIST was only recognizes as a distinct entity in 1983 when it was determined that this group of tumors contains both smooth muscles and neural features. ${ }^{2}$ GISTs originate from the GI pacemaker cells called "Interstitial cells of Cajal"3 that normally serve to regulate peristalsis. About $90 \%$ of GISTs over express KIT receptor tyrosine kinase and about $80 \%$ of GISTs show mutations of c-KIT (CD 117) gene that lead to subsequent KIT receptor activation. ${ }^{4}$ It is the varying tyrosine kinase activity that creates the wide clinical spectrum of GISTs ranging from benign to malignant tumors. ${ }^{5}$ The commonest site of origin for GIST is the stomach $(50 \%)$ followed by small intestine in around $30 \%$ of cases. ${ }^{6}$ However, GIST can involves other parts like colon \& rectum (10$15 \%)$, peritoneum \& mesentery $(<10 \%)$, \& esophagus $(<5 \%){ }^{7}$ Recently, the emergence of the new KIT tyrosine kinase inhibitor "Imatinib Mesylate" as a systemic targeted therapy showed promising results especially in advanced disease. ${ }^{8}$ Nevertheless, the treatment of choice for localized primary GIST is still the complete surgical resection with clear margins. ${ }^{9}$ Large margins are usually not needed as these tumors tend to grow out of the primary organ instead 
of diffusely infiltrating. Also, lymph node dissection is usually not needed because nodal involvement is rare. ${ }^{7}$ So, wedge resection of the stomach or segmental small intestinal resection and anastomosis is the adequate surgical approach ${ }^{10}$ that can be achieved by the classic open surgery or recently by the minimally invasive laparoscopic surgery. The later has been widely adopted as its outcomes have been shown to be comparable to the open approach in terms of blood loss, operative time, post-operative recovery, and the long term oncologic results. ${ }^{11,12}$ We conducted this study to assess the efficacy and safety of the laparoscopic resection of GIST.

\section{Patients and methods:}

This study was carried out between February 2011 \& May 2013. It included retrospective analysis of 15 patients who were submitted to laparoscopic resection of gastro-intestinal stromal tumors that were proved by histopathological examination of the removed specimen. Exclusion criteria included tumors larger than $5 \mathrm{~cm}$ and GISTs in other parts of gastrointestinal tract rather than stomach and small intestine e.g. Colon, rectum, duodenum, and esophagus.

Pre-operative investigations included laboratory tests in the form of blood count, serum electrolytes, kidney and liver function tests. Radiological investigations were done in the form of abdominal CT scan for all the patients. Upper GI endoscopy was done for gastric lesions. However, pre-operative pathological diagnosis of the GIST was not necessary to be proved prior to surgery.

The procedure was thoroughly explained to the patients and informed consent was taken from each of them.

Under general anesthesia, the patient was placed in supine position and the surgeon stood between the legs of the patient. A 30 degrees scope was inserted through the $10 \mathrm{~mm}$ umbilical port. Then, a $5 \mathrm{~mm}$ port was inserted in mid-clavicular line on both right and left sides slightly above the level of the umbilicus. A fourth $12 \mathrm{~mm}$ port was placed in the right anterior axillary line for the introduction of the endo-GIA stapler
(Covidien, USA).

Laparoscopic exploration was completely done. The small intestinal GIST Figure (1) was treated by laparoscopic resection anastomosis. While the gastric GIST Figure (2) was treated by excision with safety margins in the form of wedge resection Figure (3) or sleeve gastrectomy Figure (4). All attempts were done to avoid rupture of the tumor during the procedure. The specimen was retrieved in end-bag and sent for histopathological examination and CD117 immunohistochemistry confirmation.

Post-operative outcomes were recorded in the form of duration of parenteral analgesia, time to tolerate oral intake, and length of post-operative hospital stay.

Post-operative complications were also recorded including post-operative ileus, leakage, chest infections, and wound related complications.

All the patients were followed up each month in the first 3 months and then every 3 months with a follow up abdominal CT scan done at 6 months, 1 year, and 2 years post-operatively.

\section{Results:}

This study was conducted on 15 patients submitted to laparoscopic resection of GIST. The age of the patients ranged between 37 \& 63 years with a mean of 49 years. Eight patients $(53 \%)$ were males and $7(47 \%)$ were females.

The main presenting symptoms Table (1) were abdominal pain, nausea \& vomiting, anemic symptoms e.g. Easy fatigability, GI bleeding (haematemesis \&/or vomiting), and weight loss.

In 9 patients, the GIST was found in the stomach $(60 \%)$ and in 6 patients $(40 \%)$; it was in the small intestine as proved by preoperative diagnostic tools in the form of abdominal CT scan and upper GI endoscopy.

Laparoscopic resection was attempted in all the patients. The size of the gastric GIST ranged between $2.2 \mathrm{~cm}$ and $5 \mathrm{~cm}$ with a mean of $4.4 \mathrm{~cm}$ while the small intestinal GIST size ranged between $3.2 \mathrm{~cm}$ and 4.8 $\mathrm{cm}$ with a mean of $4 \mathrm{~cm}$. All small intestinal 


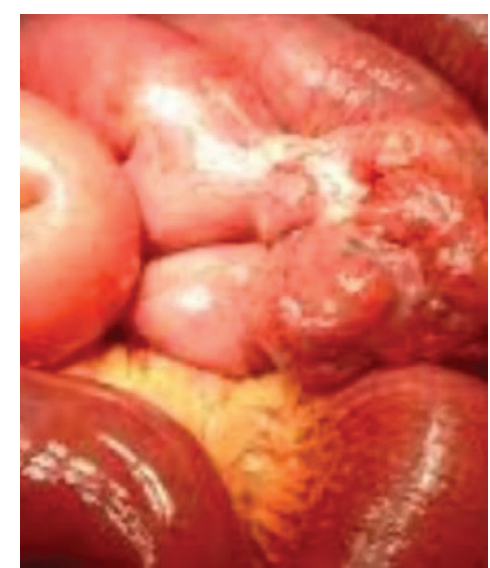

Figure (1): Small intestinal GIST on the mesenteric border of the small intestine.

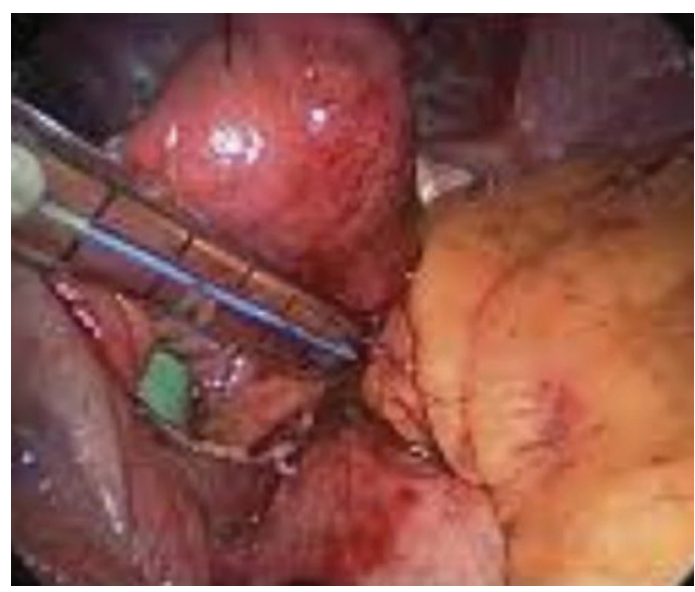

Figure (3): Wedge resection for gastric GIST.

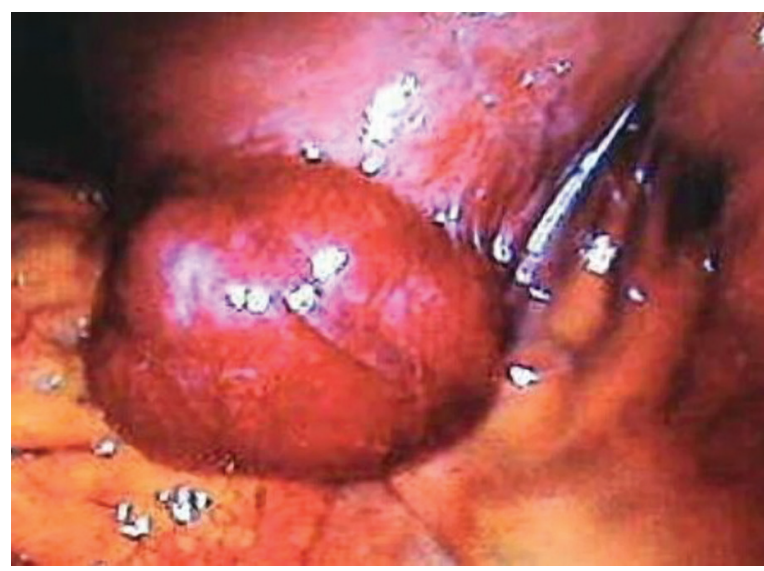

Figure (2): Gastric GIST on the greater curvature of the stomach.

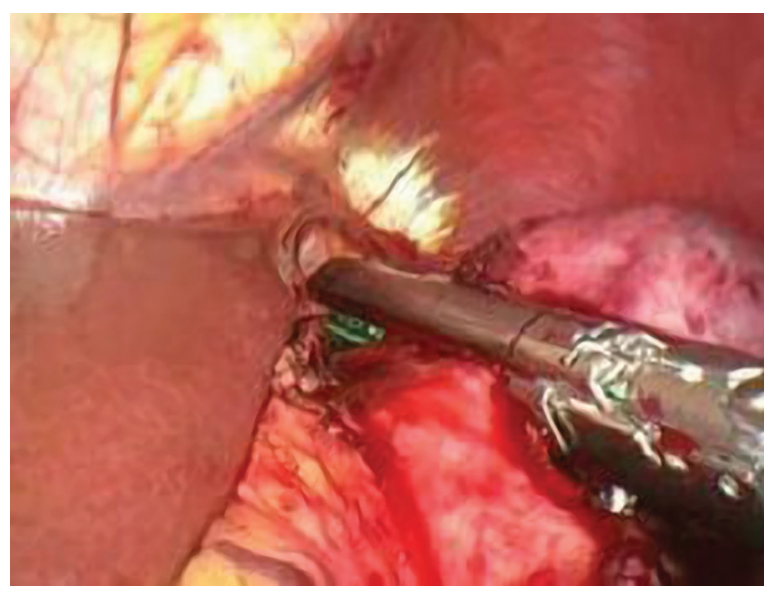

Figure (4): Sleeve gastrectomy for gastric GIST.

Table 1. Presenting Symptoms:

\begin{tabular}{|l|l|}
\hline Symptoms & No. $(\mathbf{\%})$ \\
\hline Abdominal Pain & $12(80)$ \\
\hline Anemia & $11(73)$ \\
\hline Weight Loss & $7(47)$ \\
\hline Nausea/Vomiting & $8(53)$ \\
\hline
\end{tabular}

Table 2. Risk Classification:

\begin{tabular}{|l|l|}
\hline Risk Category & No. (\%) \\
\hline Very low & $8(53 \%)$ \\
\hline Low & $4(27)$ \\
\hline Intermediate & $1(7)$ \\
\hline High & $2(13)$ \\
\hline
\end{tabular}

GIST were treated by laparoscopic resection anastomosis. Six out of the gastric GIST were treated by laparoscopic resection while
3 gastric GIST were treated by laparoscopic sleeve gastrectomies. Conversion to midline laparotomy was done in 1 case gastric GIST 
(6.7\%) to control bleeding occurred from the short gastric vessels that was injured during laparoscopic sleeve gastrectomy.

Apart from this short gastric vessels injury, no other operative complications were recorded and no tumor rupture was encountered.

Operative time ranged between 136 minutes and 98 minutes with a mean of 118.4 minutes.

Histopathological examination confirmed the diagnosis of GIST. The margins of excision were negative in all the cases with no involved margins (R0 resection). The risk classification according to The National Institute of Health Risk Criteria ${ }^{13}$ was shown in the Table (2).

Post-operatively, time to pass flatus ranged between 1 and 4 days with a mean of 1.6 days. Tolerance of full oral intake ranged between 3 and 6 days with a mean of 3.8 days. Number of doses of narcotics needed for pain control in our patients ranged between 2 and 6 days with a mean of 2.37. Length of post-operative hospital stay ranged between 4 and 8 days with a mean of 4.9 days.

Post-operative complications included 1 patient $(6.7 \%)$ with post-operative fever due to atelectasis, and 1 patient $(6.7 \%)$ with superficial infection of the umbilical wound that was treated by antibiotics and dressings. No post-operative ileus or leakage was recorded. No mortality was encountered in any of the patients.

The follow up period ranged between 24 and 3 months with a mean of 12.6 months. No recurrence or metastasis was encountered in any of the patients during follow up.

\section{Discussion:}

In 1998, Hirota and his colleagues discovered the KIT proto-oncogene mutations in GIST. It was an important step in understanding the biological behavior of this group of tumors and it led to the development of Imatinib mesylate therapy. ${ }^{14}$ Nevertheless, surgery remained always the mainstay of treatment for GIST. ${ }^{15}$ National Comprehensive Cancer Network (NCCN) guidelines recommended $\mathrm{R} 0$ resection that means tumor resection with intact pseudocapsule and negative microscopic margins for tumors $>2 \mathrm{~cm}$. NCCN guidelines also support laparoscopic resection of GIST ${ }^{16}$ as it gives the patient shorter hospital stay and less morbidity.

In our study, $53 \%$ of patients were males and $47 \%$ were females with a mean age of 49 years. In general, GIST occurs with similar frequency in both sexes. However, some studies suggest male predominance with a median age of 60 years at diagnosis. ${ }^{17}$ The main presenting manifestations of GIST in our patients were abdominal pain, anemia, nausea, vomiting, GI bleeding, and weight loss which are in accordance with many other studies $^{7,18}$. Gist was found in the stomach in $60 \%$ of patients and in small intestine in $40 \%$ of them. Other reports support these finding. However, GIST can occur in any part along the gastro-intestinal tract, but stomach and small intestine remain the commonest sites of involvement. ${ }^{7,} 19$

We used CT scan and upper GI endoscopy for pre-operative assessment of the tumors. $\mathrm{Ct}$ scan is the imaging modality of choice in GIST as it helps to localize the tumor, evaluate its extent, and detect metastasis. MRI can be helpful for tumors in fixed organs e.g. Rectum and liver. ${ }^{1}$ Standard upper GI endoscopy is useful to determine gastric tumor location and size. ${ }^{20}$ However, endoscopic biopsy is of low diagnostic yield (17-42\%) as most of the tumors are covered by normal mucosa. ${ }^{21}$ This yield can be significantly increased by the use of endoscopic ultrasound guided biopsy with a sensitivity of $78.4 \% .22$ For small intestinal GIST, enteroscopy can be used. However, it also has a low diagnostic yield as it is technically demanding and the tumor is usually growing in an extra-luminal fashion. ${ }^{23}$

In our study, the mean size of gastric GIST was $4.4 \mathrm{~cm}$ and the mean size of small intestinal GIST was $4 \mathrm{~cm}$. We excluded tumors larger than $5 \mathrm{~cm}$. NCCN guidelines originally recommended laparoscopic resection for GISTs less than $2 \mathrm{~cm}$. However, these guidelines were modified in 2007 to state that tumors up to $5 \mathrm{~cm}$ in size could be safely 
resected laparoscopically. ${ }^{16}$ Many recent studies reported laparoscopic resection for GISTs up to or even larger than $10 \mathrm{~cm} .{ }^{15,24,25}$ Large tumors may obscure the proper surgical exposure and increase the risk of tumor rupture due to their poor visualization and limited gentle dissection. Moreover, large tumors need a large laparotomy wound to retrieve the surgical specimen. ${ }^{26}$ Kim et $\mathrm{al}^{27}$ in their study suggested that with large tumor size, the repeated passage of the instruments and the increased tumor manipulations may lead to tumor exfoliation which could induce post-operative recurrence. So, they recommended doing laparoscopic resection for tumors not larger than $5 \mathrm{~cm}$ as confirmed by the pre-operative imaging studies.

We could resect the tumor laparoscopically in 14 patients. In only 1 patient $(6.7 \%)$ conversion to midline laparotomy to control bleeding from short gastric vessels was needed. No tumor rupture was encountered. The margins of excision were negative in all the patients. The mean operative time was 118.4 minutes. These results are in accordance with results of many other studies. Chen et $\mathrm{al}^{26}$ in their series had a mean operative time of 127.44 minutes with a conversion rate of $6.25 \%$. Goh et al ${ }^{28}$ reported operative time of 150 minutes and a conversion rate of $7 \%$. Although one of the goals of surgery is to obtain negative microscopic margins, wide margins as those needed for adenocarcinoma are not needed for GIST. Lymphadenectomy is also not indicated as lymph node metastases are rare. ${ }^{29}$ According to NCCN guidelines, ${ }^{30}$ tumor rupture is considered an adverse prognostic factor in GIST and patients are at very high risk of recurrence and should be discussed with the medical oncologist for possibility of adjuvant imatinib therapy.

As yet, there is no universal staging system for GIST. In general, risk of recurrence is defined according to tumor size and the mitotic count. 31,32 Gastric GISTs tend to have a more favorable prognosis than small intestinal GISTs. ${ }^{3}$ in 2002, National Institute of Health (NIH) proposed prognostic criteria for risk stratification of GIST. ${ }^{34}$ They classified GIST into very low risk (less than $2 \mathrm{~cm}$ with less than 5/50 HPF mitotic count), low risk (2-5 $\mathrm{cm}$ with less than 5/50 HPF mitotic count), intermediate risk (less than 5 cm with 6-10/50 HPF mitotic count or 5-10 cm with less than 5/50 HPF mitotic count), and high risk (more than $5 \mathrm{~cm}$ with more than 5/50 HPF mitotic count, or more than $10 \mathrm{~cm}$ with any mitotic rate, or any size with more than 10/50 HPF mitotic count). This risk stratification is essential, not only in guiding the clinician for the frequency and intensity of post-operative surveillance, but also to enable better selection of tumors for potential adjuvant therapy. Overall 5-year survival rate was found to be $100 \%$ for very low and low risk groups, $84 \%$ for the intermediate risk group, and $49 \%$ for the high risk group. ${ }^{13}$ In our series, $53 \%$ of patients were very low risk, $26 \%$ were low risk, $7 \%$ were intermediate risk, and $13 \%$ were high risk patients.

In the present study, the mean time to tolerate full oral intake was 3.8 days and mean number of narcotic doses for pain control was 2.73. The mean length of hospital stay was 4.9 days. Post-operative complications included only 1 patient with atelectasis and 1 patient with superficial wound infection. No recurrence or metastasis was encountered during a mean follow up period of 12.6 months. These results are supported by results of other studies that showed that laparoscopic resection of GIST is oncologically feasible technique that gives the patient all the advantages of minimally invasive surgery e.g. Earlier return of bowel functions, decreased duration of analgesia, shorter hospital stay, and faster return to work. ${ }^{26,28}$

In conclusion, laparoscopic resection of GIST is a safe and feasible technique that is associated with favorable perioperative results without affecting the oncological outcomes as lymphadenectomy is not needed even with the malignant variant of GIST. However, patient selection is crucial for the success of laparoscopic technique. Longer follow up is needed for further assessment of the oncological results of laparoscopic resection of GIST. 


\section{Reference:}

1- Blanke E, Eisenberg BL, \& Heinrich M: Epidemiology of GIST. Am J Gastroenterol 2005; 100: 2366-2371.

2- Mazur MT, Clark HB: Gastric stromal tumors: Reappraisal of histogenesis. $A m J$ Surg Pathol 1983; 7: 507-519.

3- Lux ML, Rubin BP, Biase TL, et al: KIT extracellular and kinase domain mutations in gastro-intestinal stromal tumors. Am J Pathol 2000; 156: 791-795.

4- Heinrich MC, Corless CL, Duensing A, et al: PDGFRA activating mutations in gastrointestinal stromal tumors. Science 2003; 299: 708-710.

5- Mucciarini C, Rossi G, Bertolini F, et al: Incidence and clinic-pathological features of gastro-intestinal stromal tumors. A population-based study. BMC Cancer 2007; 230: 1186-1190.

6- Emory TS, Sobin LH, Lukes L, et al: Prognosis of Gastro-intestinal smooth muscle (stromal) tumors: Dependence on anatomic site. Am J Surg Pathol 1999; 23: 82-87.

7- DeMatteo RP, Lewis JJ, Leung D, et al: Two hundred gastro-intestinal stromal tumors: Recurrence patterns and prognostic factors for survival. Ann Surg 2000; 231: 51-58.

8- Joensuu H, Fletcher C, Dimitrijevic S, et al: Management of malignant gastro-intestinal stromal tumors. Lancet Oncol 2002; 3: 655-664.

9- Novitsky YW, Kercher KW, Sing RF, et al: Long term outcomes of laparoscopic resection of gastric gastro-intestinal stromal tumors. Ann Surg 2006; 243: 738-747.

10- Nguyen SQ, Divino CM, Wang JL, Dikman SH: Laparoscopic management of gastrointestinal stromal tumors. Surg Endosc 2006; 20: 713-716.

11- Nishimura J, Nakajima K, Omori T, et al: Surgical strategy for gastric gastro-intestinal stromal tumors: Laparoscopic vs. Open resection. Surg Endosc 2007; 21: 875-878.

12- Choi SM, Kim Mc, Jung GJ, et al: Laparoscopic wedge resection for gastric GIST: Long-term follow up results. Eur $J$ Surg Oncol 2007; 33: 444-447.

13- Goh BK, Chow PK, Yap WM, et al: Which is the optimal risk stratification system for surgically treated localized primary GIST? Comparison of three contemporary prognostic criteria in 171 tumors and a proposal of a modified Armed Forces Institute of Pathology risk criteria. Ann Surg
Oncol 2008; 15: 2153-2163.

14- Hirota S, Isozaki K, Moriyama Y, et al: Gainof-function mutations of c-KIT in human gastro-intestinal stromal tumors. Science 1998; 279: 577-580.

15- Otani Y, Furukawa T, Yoshida M, et al: Operative indications for relatively small $(2-5 \mathrm{~cm})$ gastro-intestinal stromal tumors of the stomach based on analysis of 60 operated cases. Surgery 2006; 139: 484-492.

16- Demetri GD, Benjamin RS, Blanke CD, et al: NCCN Task Force. NCCN Task Force Report: Management of patients with gastrointestinal stromal tumor (GIST)-Update of the NCCN clinical practice guidelines. $J$ Natl Compr Canc Netw 2007; 5(2): 1-29; quiz S30.

17- Shinomura Y, Kinoshita K, Tsutsui S, Hirota $\mathrm{S}$ : Pathophysiology, diagnosis, and treatment of gastro-intestinal stromal tumors. $J$ Gastroenterol 2005; 40: 775-780.

18- Perez EA, Livingstone AS, Franceschi D, et al: Current incidence and outcomes of gastrointestinal mesenchymal tumors including gastr0intestinal stromal tumors. $\mathrm{J} \mathrm{Am} \mathrm{Coll}$ Surg 2006; 202: 623-629.

19- Mittinen M, Makhlouf H, Sobin LH, et al: Gastro-intestinal stromal tumors of jejunum and ileum: A cinico-pathologic, immunehistochemical, and molecular genetic study of 906 cases before imatinib with long term follows up. Am J Surg Pathol 2006; 30: 477-489.

20- Mullady DK, Benjamin RT: A multidisciplinary approach to the diagnosis and treatment of gastrointestinal stromal tumor. J Clin Gastroenterol 2013; 47(7): 578-585.

21- Cantor MJ, Davila RE, Faigel DO: Yield of tissue sampling for sub-epithelial lesions evaluated by EUS. Gstrointest Endosc 2003; 57: 68-72.

22- Okubo K, Yamao K, Nakamura $T$, et al: Endoscopic ultrasound-guided fine needle aspiration biopsy for the diagnosis of gastrointestinal stromal tumors in the stomach. $J$ Gastroenterol 2004; 39: 747-753.

23- Sakra L, Siller J, Vyhnalek P, \& Hacova M: Surgical treatment of gastric and small bowel gastro-intestinal stromal tumors. Videosurg 2011; 6(3): 138-143.

24- Catena F, Di Battista M, Fusaroli P, et al: Laparoscopic treatment of gastric GIST: Report of 21 cases and literature's review. $J$ Gastroentist Surg 2008; 12: 561-568.

25- Sokolich J, Galanopoulos C, Dunn E, et al: 
Expanding the indications for laparoscopic gastric resection for gastro-intestinal stromal tumors. JSLS 2009; 13: 165-169.

26- Chen YH,Liu KH, Yeh CN, etal:Laparoscopic resection of gastro-intestinal stromal tumors: Safe, efficient, and comparable oncologic outcomes. J Laparoendesc \& Advanced Surg Tech 2012; 22(8): 758-763.

27- Kim MD, Kang DH, Park JH, et al: Abdominal wound metastasis after laparoscopic surgery of gastro-intestinal stromal tumor. Gut Liver 2010; 4: 283-286.

28- Goh BKP, Chow PKH, Chok AY, et al: Impact of the introduction of laparoscopic wedge resection as a surgical option for suspected small/medium-sized gastro-intestinal stromal tumors of the stomach on perioperative and oncologic outcomes. World J Surg 2010; 34: 1847-1852.

29- Fong Y, Coit DG, Woodruff JM, et al: Lymph node metastasis from soft tissue sarcoma in adults. Analysis of data from a prospective database of 1772 sarcoma patients. Ann Surg 1993; 217: 72-77.
30- Rutkowski P, Bylina E, Wozniak A, et al: Validation of the Joensuu risk criteria for primary resectable gastro-intestinal stromal tumor-the impact of tumor rupture on patient outcomes. Eur J Surg Oncol 2011; 37: 890-896.

31- Bucher P, Egger JF, Gervaz P, et al: An audit of surgical management of gastro-intestinal stromal tumors (GIST). Eur J Surg Oncol 2006; 32: 310-314.

32- Gupta M, Sheppard BC, Corless CL, et al: Outcome following surgical therapy for gastro-intestinal stromal tumors. $J$ Gastrointest Surg 2006; 10: 1099-1105.

33- Avanzato RD, Carboni F, Palmieri MB, et al: Laparoscopic resection of sporadic synchronous gastric and jejunal gastrointestinal stromal tumors: Report of a case. Surg Today 2009; 39: 335-339.

34- Fletcher CD, Berman JJ, Corless C, et al: Diagnosis of gastro-intestinal stromal tumors: A consensus approach. Hum. Pathol 2002; 33: 459-465. 


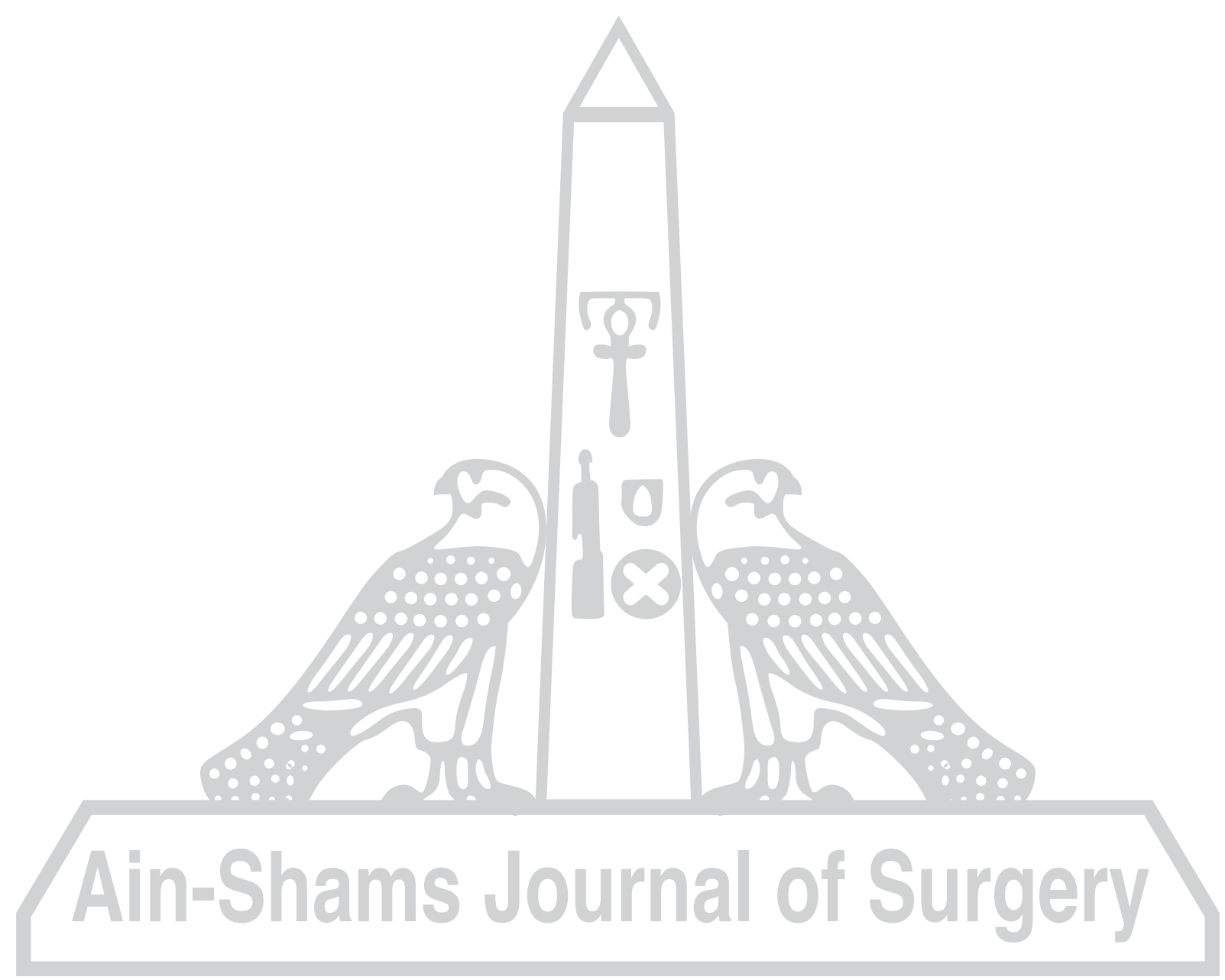

